\title{
Urinary schistosomiasis among preschool children in Malengachanzi, Nkhotakota District, Malawi: Prevalence and risk factors
}

\author{
VB Moyo ${ }^{1}$,W Changadeya ${ }^{*}$, S Chiotha ${ }^{1}$, D Sikawa ${ }^{2}$
}

1. Master of Environmental Sciences Programme, Faculty of Sciences, Chancellor College, University of Malawi, Zomba, Malawi

2. Department of Aquaculture and Fisheries Science, Lilongwe University of Agriculture and Natural Resources, Lilongwe, Malawi

Correspondence can be addressed: wchanga@cc.ac.mw

\begin{abstract}
Aim

This study was designed to determine the prevalence of and risk factors for schistosomiasis among a group of preschool children in Malawi. Schistosomiasis burden among preschoolers in Malawi is not well documented in the literature.

\section{Methods}

This study used field research (in the form of a snail survey), laboratory work (urinalysis and microscopy for parasite identification), and questionnaireguided interviews to determine the prevalence of and risk factors for urinary schistosomiasis among children, aged between 6 and 60 months, in Malengachanzi, Nkhotakota District, Malawi.

\section{Results}

Urinary schistosomiasis prevalence among preschool children was $13 \%$. Of the factors evaluated, only age $(\mathrm{P}=0.027)$ was statistically significantly associated with urinary schistosomiasis risk. Four-year-old preschool children were five times more likely to contract urinary schistosomiasis than twoyear-old children (odds ratio $[\mathrm{OR}]=5.255 ; 95 \%$ confidence interval $[\mathrm{CI}]=1.014-27.237 ; \mathrm{P}=0.048$ ). Increased contact with infested water among older children likely explains much of their increased risk. Infestation was evidenced by the presence of infected Bulinus globosus snails in the water contact points surveyed. Multiple regression analysis showed that visiting water contact sites daily $(\mathrm{OR}=0.898,95 \% \mathrm{CI}=0.185-4.350, \mathrm{P}=0.894)$, bathing in these sites $(\mathrm{OR}=9.462,95 \% \mathrm{CI}=0.036-0.00, \mathrm{P}=0.430)$ and lack of knowledge, among caregivers, regarding the causes of urinary schistosomiasis $(\mathrm{OR}$ $=0.235,95 \% \mathrm{CI}=0.005-1.102, \mathrm{P}=0.066)$ posed statistically insignificant risk increases for preschoolers contracting urinary schistosomiasis.
\end{abstract}

\section{Conclusions}

Urinary schistosomiasis was prevalent among preschool children in Malengachanzi, Nkhotakota District. Contact with infested water puts these children and the general population at risk of infection and reinfection. Inclusion of preschool children in treatment programmes should be considered imperative, along with safe treatment guidelines. To prevent infection, the population in the area should be provided with health education and safe alternative water sources.

\section{Introduction}

Schistosomiasis, also commonly known as bilharzia, is a waterborne parasitic infection most common in rural areas of developing countries. ${ }^{1,23}$ Estimates show that 200 million people worldwide are at risk of infection, ${ }^{4}$ while 120 million people already display symptoms of the disease. ${ }^{5}$ It has been estimated that more than $97 \%$ of all schistosomiasis cases occur in Africa, ${ }^{6,7}$ and high prevalence estimates and infection intensities are found in school-aged children, adolescents, and adults. ${ }^{1,8,9}$ In order for schistosomes to complete their lifecycles, they require aquatic intermediate snail hosts. Thus, the distribution of susceptible populations of Bulinus and Biomphalaria species broadly outlines endemic areas where both urinary and intestinal schistosomiasis occurs. ${ }^{10,11}$

There are two major forms of schistosomiasis prevalent in Malawi, the urinary form, caused by Schistosoma haematobium, and the intestinal form, caused by Schistosoma mansoni. ${ }^{3,10}$ Urinary schistosomiasis is a public health problem that affects the general health, as well as the cognitive development of children. ${ }^{11-16}$ Estimates of its prevalence vary across Malawi, ${ }^{17}$ with some estimates suggesting that $40 \%$ to $50 \%$ of Malawians are infected. ${ }^{2,9,18}$ It has been suggested that these estimates were derived from studies conducted too long ago (in the 1990s) to be valid now, and that they were http://dx.doi.org/10.4314/mmj.v28i1.3 skewed by a selection bias for high-risk areas. ${ }^{2,9}$ This suggests that more up-to-date local estimates would be more helpful for planning of control strategies.

In Malawi, schistosomiasis in infants and preschool children has been documented in recent years, revealing an important demographic that was previously overlooked in the public health context of schistosomiasis. ${ }^{6,7,11}$ For instance, lack of a suitable paediatric praziquantel formulation has excluded preschool children from mass drug administration efforts. Preschool children have been overlooked despite emerging evidence from other countries and other parts of Malawi that preschoolers carry significant levels of schistosomiasis infection and are at risk of infection just like their older school-aged counterparts. ${ }^{12,21,23}$ No study in Nkhotakota District has focused on urinary schistosomiasis prevalence and risk factors among preschool children.

In order to estimate the burden of the disease, it remains important to collect up-to-date information to assist in planning treatment and prevention strategies. Therefore, this study aimed at providing prevalence estimates for preschool children in the Malengachanzi area of Nkhotakota, in order to provide updated epidemiological information to inform treatment strategies in the district. In addition, the focus of this study extended to assessing the risk factors for this age 
group, including the presence of intermediate snail hosts. Consequently, efforts to control the disease and to develop adequate care initiatives for this age group can be informed by the necessary baseline information.

\section{Methods}

\section{Study area}

The study was undertaken in Traditional Authority (TA) Malengachanzi, in the lakeshore district of Nkhotakota, which is in Malawi's Central Region. The district is located on the west coast of Lake Malawi. Four villages were purposively selected for the study, since they are impact areas for the Ecological Health Project, of which this study was a component. These villages were Chiula, Makuzi, Kalimanjira, and Kalusa.

\section{Study design}

This was a cross-sectional study that involved three distinct methods of data collection, namely field research (a snail survey), a laboratory-based parasitological survey (urinalysis and urine microscopy for blood detection and parasite identification), and questionnaire-guided interviews of guardians and caregivers of the children. The field phase of the data collection was carried out between August and September 2012.

\section{Snail survey}

Prior to the parasitological survey, each village was visited to inspect local water habitats for freshwater snails at various local water contact points. With the assistance of local knowledge and actual visitation of the sites, three water contact points were identified in the villages (Mtanga swamp, Mambala River, and Chiwovu fishing point on Chia Lagoon). Survey time was quantified, with two collectors surveying the water contact points for 1 hour per contact site, expressed in numbers per man-hour search. Gloves and protective shoes were worn by the field personnel to avoid direct contact with water and thus prevent acquisition of schistosomiasis. Collected snails were placed on a flattened plastic sheet, where they were sorted and identified. The identified Bulinus globosus snails were then counted and collected using forceps and each was placed in separate beakers. The beakers were labelled according to the water contact points where the snails were collected.

\section{Snail identification}

Identification of the snails was done according to techniques by Duwa and Oyeyi and Chitsulo et. al,. based on morphological identification keys. ${ }^{19,20,21}$ Those that were identified as Bulinus globosus were counted and the rest were thrown back into the water.

\section{Determination of infected Bulinus globosus snails}

Upon identification, random samples of 20 snails from each site were selected and tested for schistosome infection. This was done by putting each B. globosus snail in a separate beaker with distilled water and exposing them to sunlight for about three to four hours. ${ }^{10,19}$ Handheld lenses were used to observe cercariae shedding. Snails that did not shed cercariae were taken to the Chancellor College Biology Department Laboratory, where they were crushed in a petri dish using a spatula, and the presence or absence of sporocysts was determined using a microscope. ${ }^{19}$

\section{Parasitological survey Urine collection}

Sensitization for collection of urine samples was done at least two days prior to the survey, through the Village Headman, the Health Surveillance Assistant (HSA), and the Village Health Committee. A total of 143 preschool children were enumerated in the community in a house-to-house census conducted for children aged between 6 and 60 months. The small number of preschool children in the area allowed for the inclusion of all children in the study area who were within the specified age range. None of the prospective participants refused to take part in the study. Labelled plastic containers were given to parents and guardians of the children to collect urine samples. Each bottle of urine was labelled to correspond to the participants' questionnaire numbers. Urine collection was done between the hours of 10:00 a.m. and 2:00 p.m.. The urine specimens were immediately taken to Nkhotakota District Hospital Laboratory for analysis by the district laboratory technician.

\section{Examination of urine for microhaematuria}

All urine specimens were examined for infection with Schistosoma haematobium, by first determining the presence of blood in the urine. Urinalysis reagent strips (Cortez Diagnostics Inc., Woodland Hills, CA, USA) were used to detect the presence of haemoglobin in the urine, following methods described by Ekpo et al. ${ }^{21}$ The colours found on the strips were directly compared to the colours indicated on the reagent strip bottle in order to determine the exact category of the colour. The colour values were labelled as negative $(-)$, slightly positive $(+)$, positive $(++)$, and strongly positive $(+++)$, each corresponding to the amount of blood found in the urine sample being tested.

\section{Examination for Schistosoma haematobium ova}

A total of $10 \mathrm{~mL}$ of each urine sample was centrifuged at $5000 \mathrm{rpm}$ for five minutes, following methods described by Ekpo et al. ${ }^{21}$ After centrifugation, the supernatant was discarded, and the remaining sediment was transferred to the centre of a clean glass slide and covered with a cover slip. Each prepared slide was mounted on a microscope and examined at x40 magnitude to identify Schistosoma haemotobium ova, which are characterized by a terminal spine. The eggs were counted and recorded as number of eggs/10 mL of urine.

\section{Questionnaire-guided interviews}

Each child's guardian or caregiver was interviewed using a standardized questionnaire. The questionnaire was formulated to capture information on sociodemographic factors (age, sex), education levels, water contact patterns, sanitation, history of infection, water sources, and knowledge and awareness of the disease. This was done in order to assess risk factors associated with urinary schistosomiasis infection. Only parents whose children submitted urine samples were eligible for the questionnaire interview. A total of 77 guardians were interviewed in this study. Some of them represented more than one child, hence a smaller number of them than that of total preschool children registered.

\section{Data analysis}

The mean abundance of snails was also analyzed using the independent t-test. Data from the questionnaire survey were analyzed using SPSS version 16.0. Differences in proportions of preschool children were tested using the chi-square test. 
The numbers of eggs counted were transformed to $\log 10$ $(x+1)$ values to normalize the distribution of the residual values for statistical analyses. Multiple logistic regression analysis was done to determine the odds ratio (OR) for each of the examined risk factors for urinary schistosomiasis.

\section{Ethical considerations}

Ethical clearance was granted by the Malawi Ministry of Health, through the National Health Sciences Research Committee (NHRSC). Local support was given by Nkhotakota District Health Office and the village chiefs. In each village, the Village Headman, together with guardians and caregivers were fully briefed on the objectives of the study, with assistance from the HSA of the area. Thereafter, assent and written informed consent was obtained from each child and guardian, respectively. Any preschoolaged children who were detected to have haematuria by reagent strips (and were thus presumptively diagnosed with schistosomiasis),were treated on-site with praziquantel (40 $\mathrm{mg} / \mathrm{kg}$ ). For accurate dosing, weight was measured using a digital scale for each patient. Younger children were provided with crushed tablets. After treatment, children were observed by a nurse for two to three hours to monitor for immediate complications.

\section{Results}

\section{Presence, abundance, and infection of Bulinus globosus snails at water contact points}

All water contact points harboured Bulinus globosus snails (Table 1). The abundance per catch (search hour) figures at each site were as follows: 64 snails at Chiwovu, 42 at Mtanga, and 21 at Mambala. The abundance was based on the limited one-hour search that was allotted to each site. Analysis of vector snails showed that, on average, $71 \%$ of the Bulinus globosus snails were infected with cercariae. Mtanga had the highest cercariae shedding (85\%), followed by Mambala $(80 \%)$, and finally Chiwovu (65\%) (Table 1).

Table 1: Abundance and infection status of Bulinus globosus snails in Malengachanzi area

\begin{tabular}{lcccc} 
Water contact point & $\begin{array}{c}\text { No. of enails } \\
\text { collected per } \\
\text { hour }\end{array}$ & $\begin{array}{c}\text { No. (\%) of } \\
\text { snails shedding } \\
\text { cercariae } \\
\text { (out of 20) }\end{array}$ & $\begin{array}{c}\text { No. (\%) of } \\
\text { sporocyst- } \\
\text { positive snails } \\
\text { (out of 20) }\end{array}$ & $\begin{array}{c}\text { No. (\%) of } \\
\text { infected } \\
\text { snails } \\
\text { (out of 20) }\end{array}$ \\
\hline Mtanga (Chia Lagoon) & 42 & $9(45)$ & $8(40)$ & $17(85)$ \\
Tambala River & 64 & $9(45)$ & $4(20)$ & $13(65)$ \\
Chiwovu (Chia Lagoon) & 21 & $14(70)$ & $2(10)$ & $16(80)$ \\
\hline
\end{tabular}

\section{Prevalence and intensity of urinary schistosomiasis in Malengachanzi}

The mean age of preschool children enrolled in the study was 3.77 years. Four-year-olds, who made up $34.3 \%$ of the participants, comprised the largest birth year cohort, while children between six months and one year of age $(3.5 \%)$ were the smallest cohort. Sixty-four percent of the participants were boys.

Eighteen preschool children $(13 \%)$ tested positive for $S$. baematobium infection (Table 2), on the basis of centrifuged urine microscopy. Out of 51 female preschool children examined, $12 \%$ had evidence of Schistosoma haematobium in their urine, while among the 92 male preschool children examined, about $13 \%$ had positive urine. With regards to age, the highest prevalence $(32 \%)$ was found in the four-year- olds. Prevalence gradually diminished with younger age: $12 \%$ of three-year-olds and $6 \%$ of two-year-olds had evidence of S.haematobium infection. Male and female preschool children aged less than two years had no overt evidence of urinary schistosomiasis infection.

Eleven children $(7.7 \%)$ tested positive for microhaematuria. At $16.4 \%$ ( $\mathrm{n}=9$ ), the four-year-olds had the highest prevalence of microhaematuria. There was no statistically significant association between microhaematuria and age $(\mathrm{P}$ $=0.526$ ).

Table 2: Prevalence and intensity of urinary schistosomiasis among preschool children in Malengachanzi area

\begin{tabular}{|c|c|c|c|c|c|c|}
\hline \multirow[t]{2}{*}{$\begin{array}{l}\text { Demographic } \\
\text { characteristics }\end{array}$} & \multicolumn{6}{|c|}{ Frequency (\%) } \\
\hline & Total & $\begin{array}{c}\text { Microscopic } \\
\text { evidence of } S \text {. } \\
\text { baematobitam in } \\
\text { urine }\end{array}$ & $\begin{array}{c}\text { Microhaematuria } \\
\text { present }\end{array}$ & $\begin{array}{c}\leq 10 \\
\text { eggs/mL } \\
\text { in urine }\end{array}$ & $\begin{array}{l}11 \text { to } 30 \\
\text { eggs/mL } \\
\text { in urine }\end{array}$ & $\begin{array}{l}31 \text { to } 50 \\
\text { eggs } / \mathrm{mL} \\
\text { in urine }\end{array}$ \\
\hline Total & 143 & $18(13)$ & $11(7.7)$ & $10(58)$ & $6(33)$ & $2(9)$ \\
\hline \multicolumn{7}{|l|}{ Sex } \\
\hline Male & 92 & $12(13)$ & $6(6.5)$ & $7(58)$ & $2(33)$ & $1(9)$ \\
\hline Female & 51 & $6(12)$ & $5(9.8)$ & $3(50)$ & $2(33)$ & $1(17)$ \\
\hline \multicolumn{7}{|l|}{ Age (months) } \\
\hline $6<$ age $<12$ & 5 & $0(0)$ & $0(0)$ & $0(0)$ & $0(0)$ & $0(0)$ \\
\hline $12<$ age $<24$ & 16 & $0(0)$ & $0(0)$ & $0(0)$ & $0(0)$ & $0(0)$ \\
\hline $24<$ age $<36$ & 33 & $2(6)$ & $0(0)$ & $2(100)$ & $0(0)$ & $0(0)$ \\
\hline $36<$ age $<48$ & 34 & $4(12)$ & $2(5.8)$ & $1(25)$ & $2(50)$ & $1(25)$ \\
\hline $48<$ age $<60$ & 55 & $12(22)$ & $9(16)$ & $7(58)$ & $33(60)$ & $1(9)$ \\
\hline
\end{tabular}

\section{Risk of contracting urinary schistosomiasis infection in the Malengachanzi area}

Multiple logistic regression analysis collected using the questionnaire response data (Table 3) revealed that fouryear-old preschool children had a 5.26 times higher risk of contracting urinary schistosomiasis infection compared to two-year-old preschool children (odds ratio $[\mathrm{OR}]=5.255$, $95 \%$ confidence interval $[\mathrm{CI}]=1.014-27.237, \mathrm{P}=0.048$ ). Age was the only risk factor associated with a statistically significant risk of urinary schistosomiasis acquisition. In relation to the children who were not allowed or were too young to visit the sites, the odds ratio for contracting urinary schistosomiasis was $0.898(95 \% \mathrm{CI}=0.185-4.350, \mathrm{P}$ $=0.8940)$ for preschool children who visited water contact sites daily (Table 3 ).

Table 3: Logistic regression of risk factors associated with urinary schistosomiasis among preschool children in Malengachanzi area

\begin{tabular}{lcccc}
\hline Water contact point & $\begin{array}{c}\text { No. of snails } \\
\text { collected per } \\
\text { hour }\end{array}$ & $\begin{array}{c}\text { No. (\%) of } \\
\text { snails shedding } \\
\text { cercariae } \\
\text { (out of 20) }\end{array}$ & $\begin{array}{c}\text { No. (\%) of } \\
\text { sporocyst- } \\
\text { positive snails } \\
\text { (out of 20) }\end{array}$ & $\begin{array}{c}\text { No. (\%) of } \\
\text { infected } \\
\text { snails } \\
\text { (out of 20) }\end{array}$ \\
\hline Mtanga (Chia Lagoon) & 42 & $9(45)$ & $8(40)$ & $17(85)$ \\
Mambala River & 64 & $9(45)$ & $4(20)$ & $13(65)$ \\
Chiwovu (Chia Lagoon) & 21 & $14(70)$ & $2(10)$ & $16(80)$ \\
\hline
\end{tabular}

The odds ratio associated with preschool children whose caregivers had no knowledge of the risk factors and preventive measures for urinary schistosomiasis was 0.235 (95\% CI $=0.050-1.102, \mathrm{P}=0.066)$. The study findings indicated high levels of awareness: knowledge of causation was at $71 \%$, prevention $88 \%$, and treatment $80 \%$. 


\section{Discussion}

Our findings reveal the prevalence of urinary schistosomiasis among preschool-aged children in the Malengachanzi area in Nkhotakota District. These findings have added to the growing body of evidence that preschool-aged children harbour infection with urinary schistosomiasis. They also highlight the need to evaluate schistosomiasis prevalence in preschool-aged children in other parts of Malawi, where urinary schistosomiasis is considered hyperendemic. The occurrence and severity of schistosomiasis among preschoolaged children is currently receiving increased attention because of the extent and significance of the disease in this age group, ${ }^{4}$ and the lack of adequate information about paediatric dosing and safety of praziquantel. ${ }^{11}$

The study showed significant differences in prevalence related to age. Both prevalence and intensity of infection increased with increasing age. This observation is in line with other research ${ }^{11,20}$ that revealed infection even in preschool children less than two years of age. Older children, aged between two and five years, also had evidence of infection, meaning that infection with urinary schistosomiasis occurs early in life, through exposure to contaminated water. Interestingly, the proportion of infected male preschool children (with intensity of less than 10 eggs per $10 \mathrm{~mL}$ of urine) was higher $(58 \%)$ than that for females $(50 \%)$. However, statistical testing using chi-square showed that there was no significant difference in prevalence between males and females $(\mathrm{P}=0.827)$.

The numerical difference in prevalence rate and intensity of urinary schistosomiasis between boys and girls could be attributed to frequency of contact with infested water, which was higher among boys than girls in all age groups (Table 2). To support this observation, caregivers reported that despite all the children accompanying their mothers when conducting domestic chores at various water points, male preschool children are likely to join fellow boys in swimming and playing in water than female preschool children.

Similarly, the significant increased infection rate with age can be attributed to the play habits of four-year-old children, which are similar to school-aged children. Given their relatively advanced physical and motor development, schoolaged children are more likely to engage in independent water-based play, as well as assist with chores that involve contact with water bodies. This observation implies that frequency of contact with water is an important factor in schistosomiasis prevalence. Similar observations have also been reported elsewhere. ${ }^{21,22}$ In this case, water contact habits could be the major contributor to the differences observed.

Since all the water contact points were found to be harbouring infected snails, caretakers and preschool children that contact water at these sites (Mambala, Chiwovu and Mtanga), in the course of conducting domestic chores or bathing, are at risk of contracting urinary schistosomiasis, especially because these activities take place at the shallower areas of water bodies. ${ }^{3}$

With limited sources of clean water in the community, it is difficult if not impossible to prevent the community from visiting infested sites every day for various necessities. This is because the study areas are rural communities that depend on the river, ponds, wells, and dams for their water needs and livelihoods: farming (irrigation), fishing, bathing, and other domestic uses. Bodies of water are distributed within the area, providing natural water sources and also serving as nidi for the schistosome parasites and snail intermediate hosts, where people interact during their various activities

In other areas, efforts to control the use of infested water has included provision of alternative safe sources of water that were only used for drinking by the communities. ${ }^{21}$ This suggests that provision of safe water, as well as intensification of health education programmes, coupled with suitable paediatric praziquantel information could be used to eliminate the disease among preschool children.

\section{Conclusions}

This study has established that Schistosoma haematobium is prevalent among preschool children in the Malengachanzi area in Nkhotakota District. Inclusion of preschool children in treatment programmes should be considered imperative, along with safe treatment guidelines for this age group. It can also be concluded that older preschool children have generally higher prevalence of urinary schistosomiasis compared to younger ones. Infested water points in Malengachanzi put preschool-aged children, their caregivers, and the general population at risk of contracting urinary schistosomiasis infection. Therefore, in order to avoid infection and reinfection, there is a need to provide civic education to prevent children and adults from unnecessary contact with contaminated water bodies. Further investigation of the extent of the disease among preschool children throughout the country is recommended.

\section{Acknowledgements}

We are most grateful to the Eco-Health Project Team (Malawi and Tanzania) for sponsoring this study at Chancellor College, University of Malawi. We are also indebted to the preschoolaged children of the Malengachanzi area and their parents, the Chancellor College Biology Laboratory technicians, Group Village Headman Kalimanjira, and the Nkhotakota District Health Office for their excellent cooperation in this study.

\section{References}

1. Southgate VR, Rollinson D, Tchuem Tchuenté LA, Hagan P. Towards control of schistosomiasis in sub-Saharan Africa. J Helminthol. 2005 Sep;79(3):181-5.

2. National Schistosomiasis Control Programme, Malawi. Ten year plan of action 1991-2000. Lilongwe: Ministry of Health and Population, Malawi; 1991.

3. Schistosomiasis Control Programme, Community Health Surveillance Unit 1997-2001. Lakeshore schistosomiasis control project. Lilongwe: Ministry of Health and Population, Malawi; 2001.

4. World Health Organization. Schistosomiasis: progress report 20012011 and strategic plan 2012-2020. Geneva: World Health Organization; 2013.

5. van der Werf MJ, de Vlas SJ, Brooker S, Looman CW, Nagelkerke NJ, Habbema JD, et al. Quantification of clinical morbidity associated with schistosome infection in sub-Saharan Africa. Acta Trop. 2003 May;86(2-3):125-39.

6. Stothard JR, Gabrielli AF. Response to Johansen et al.: Leave children untreated and sustain inequity! Trends Parasitol. 2007 Dec;23(12):56970 .

7. Stothard JR, Sousa-Figueiredo JC, Betson M, Bustinduy A, ReinhardRupp J. Schistosomiasis in African infants and preschool children: let them now be treated! Trends Parasitol. 2013 Apr;29(4):197-205. doi: 10.1016/j.pt.2013.02.001 
8. Woolhouse ME. Patterns in parasite epidemiology: the peak shift. Parasitol Today. 1998 Oct;14(10):428-34.

9. Makaula P, Sadalaki JR, Muula AS, Kayuni S, Jemu S, Bloch P. Schistosomiasis in Malawi: a systematic review. Parasit Vectors. 2014 Dec 10;7:570. doi: 10.1186/s13071-014-0570-y.

10. Madsen H, Stauffer JR Jr. The burrowing behaviour of Bulinus nyassanus, intermediate host of Schistosoma haematobium, in Lake Malawi. AfrJ Aquat Sci. 2012;37(1):113-6. doi:10.2989/16085914.20 12.666383 .

11. Poole H, Terlouw DJ, Naunje A, Mzembe K, Stanton M, Betson $\mathrm{M}$, et al. Schistosomiasis in pre-school-age children and their mothers in Chikhwawa district, Malawi with notes on characterization of schistosomes and snails. Parasit Vectors. 2014 Apr 1;7:153. doi: 10.1186/1756-3305-7-153

12. World Health Organization. Report of a meeting to review the results of studies on the treatment of schistosomiasis in preschool-age children. Geneva: World Health Organization; 2011.

13. Bausch D, Cline BL. The impact of control measures on urinary schistosomiasis in primary school children in northern Cameroon: a unique opportunity for controlled observations. Annual journal of tropical medicine and hygiene. Am J Trop Med Hyg. 1995 Dec;53(6):577-80.

14. Danso-Appiah A, De Vlas SJ, Bosompem KM, Habbema JD. Determinants of health seeking behaviour for schistosomiasis-related symptoms in the context of integrating schistosomiasis control within the regular health services in Ghana. Trop Med Int Health. 2004 Jul;9(7):784-94.

15. Kayuni S, Peeling R, Makaula P. Prevalence of S.haematobium infection among school children living in south-western shores of Lake Malawi [dissertation]. London: London School of Hygiene and Tropical Medicine; 2012.

16. Chipeta MG, Ngwira B, Kazembe LN. Analysis of Schistosomiasis haematobium infection prevalence and intensity in Chikhwawa,
Malawi: an application of a two part model. PLoS Negl Trop Dis. 2013;7(3):e2131. doi: 10.1371/journal.pntd.0002131..

17. Engels D, Chitsulo L, Montresor A, Savioli L. The global epidemiological situation of schistosomiasis and new approaches to control and research. Acta Trop. 2002 May;82(2):139-46.

18. Bowie C, Purcell B, Shaba B, Makaula P, Perez M. A national survey of the prevalence of schistosomiasis and soil transmitted helminths in Malawi. BMC Infect Dis. 2004 Nov 16;4:49.

19. Duwa MR, Oyeyi TI. The role of Jakara dam in the transmission of schistosomiasis. Bayero Journal of Pure and Applied Sciences. 2009;2(1):58-63.

20. Chitsulo L, Lengeler C, Jenkins J. The schistosomiasis manual: a guide for the rapid identification of communities with a high prevalence of urinary schistosomiasis. Geneva: World Health Organization; 1995 (unpublished document TDR/SER/MSR/95.2. Social and Economic Research Report Series).

21. Ekpo UF, Laja-Deile A, Oluwole AS, Sam-Wobo SO, Mafiana CF. Urinary schistosomiasis among preschool children in a rural community near Abeokuta, Nigeria. Parasit Vectors. 2010 Jul 5;3:58. doi: 10.1186/1756-3305-3-58.

22. Kapito-Tembo AP, Mwapasa V, Meshnick SR, Samanyika Y, Banda D, Bowie C, et al. Prevalence distribution and risk factors for Schistosoma haematobium infection among school children in Blantyre, Malawi. PLoS Negl Trop Dis. 2009;3(1):e361. doi: 10.1371/ journal.pntd.0000361.

23. Seto EY, Wong BK, Lu D, Zhong B. Human schistosomiasis resistance to praziquantel in China: should we be worried? Am J Trop Med Hyg. 2011 Jul;85(1):74-82. doi: 10.4269/ajtmh.2011.10-0542. 